\title{
FIN SUBJETIVO Y RAZÓN INSTRUMENTAL EN CIENCIA DE LA LÓGICA DE HEGEL
}

Subjective End and instrumental Reason by Hegel's Science of Logic

\author{
SERGIO MONTECINOS FABIO \\ Ruhr-Universität Bochum (Alemania) \\ montecinos.fabio.s@gmail.com
}

\section{Resumen}

Se sostiene que en la Ciencia de la Lógica Hegel distingue, mediante la categoría de "fin subjetivo", una dimensión puramente instrumental de la actividad del concepto. Tras delinear una lectura de conjunto de la Doctrina del Concepto se reconstruyen sectores clave del capítulo dedicado a la Teleología con vistas a detectar tanto los rasgos fundamentales como las limitaciones de esta dimensión puramente instrumental del concepto. Se concluye que es posible verificar en el capítulo una "lógica de la actividad instrumental" e interpretar en dicha clave la posición sistemática del capítulo. Además, se subrayan los rasgos críticos del tratamiento hegeliano en vista del problema general de una mecanización y desgaste del mundo y de la vida, lo que proyecta el problema al tránsito desde el capítulo Teleología a la sección Idea.

Palabras clave: Crítica de la razón instrumental; lógica de Hegel; mecanismo y finalidad (Zweckmäßigkeit); realización del concepto en la objetividad.

\section{Abstract}

This paper seeks to show that, in the Science of Logic Hegel, through the category of "subjective end", distinguishes a purely instrumental dimension of the activity of concept. After delineating a general interpretation of the Doctrine of Concept, key aspects of the chapter dedicated of Teleology are re-constructed with the aim of detecting both the fundamental features and the limitations of this purely instrumental dimension of the concept. It is concluded that, in the chapter, it is possible to verify a 'logic of the instrumentally activity' and from this standpoint, to interpret the systematic position of the chapter. In addition, critical features of the Hegelian analysis are emphasized in regard to the general problem of mechanization and wear of the world and life, which projects the problem to the transit from chapter on Teleology to the Idea section.

Key words: Critic of instrumental reason; Hegel's logic; mechanism and end (Zweckmäßigkeit); realization of the concept in objectivity.

\section{PLANTEAMIENTO}

En el tercer libro de la Ciencia de la Lógica, la Doctrina del Concepto (=DC), el tercer capítulo de la segunda sección, titulada La Objetividad, se encuentra dedicado a la exposición de la Teleología. Según el procedimiento deductivo inmanente propio de la Lógica, el pensamiento (Gendanke) implicado en la Teleología sería un momento necesario, y al mismo tiempo limitado, del desarrollo del concepto en su doctrina. En lo 
que sigue quisiéramos dar cuenta de ambos aspectos en conexión con una determinada lectura del capítulo, inserta a su vez en una interpretación general de la DC. Para plantear concretamente el asunto que nos ocupará conviene bosquejar preliminarmente esta visión global de la DC, ya que esto nos permitirá plantear más precisamente el sentido en que abordaremos el capítulo sobre la Teleología.

Como sabemos, la Doctrina de la Esencia -y con ello la entera Lógica Objetivaculmina con la determinación de la necesidad (Notwendigkeit) de la sustancia y su asunción (Aufhebung) en la libertad del concepto. De acuerdo con este movimiento final de la Doctrina de la Esencia, frente a la necesidad sustancial, la DC ha de exponer el modo de existencia libre del concepto. En primer lugar, esto implica un giro en el tipo de dialéctica que articula la exposición: la dialéctica reflexiva de la esencia da lugar a una dialéctica que es propia del concepto (Entwicklungsdialektik), consistente en ser sí mismo en lo otro (vid. Schäfer, 2001, p. 311). A su vez, implica que este "sí mismo" que es en lo otro - en el sentido de "desarrollarse" y "existir" en ello- no tiene el modo de ser del objeto sino el de la autoconsciencia: el concepto se desarrolla mediante diversas formas de reconocimiento de su hacer en lo objetivo, por cuanto lo objetivo se revela para él como puesto por su propia libertad. De ahí que Hegel distinga la ciega necesidad, propia de la sustancia, del carácter transparente del concepto en su doctrina: el concepto adquiere claridad respecto de sí mismo en las determinaciones del contenido, pues ellas no son sino la manifestación de su propia autodeterminación. En tal sentido puede afirmarse, de acuerdo con la tesis de Nuzzo (1995, 2003), que si la Lógica Objetiva expone desde su inicio el "concepto del ser" hasta consumar su esencia como sustancialidad, entonces la Lógica Subjetiva ha de exponer, por su parte, el "ser del concepto" (Nuzzo, 1995, pp. 110-111), lo que puede entenderse tanto en el sentido de que ella exhibe el modo de ser del concepto en su despliegue, como en el sentido de que ella exhibe aquello que es puesto por el concepto como ser: la objetividad mediada por su actividad.

Con lo señalado ya es posible señalar una directriz que, desde nuestra perspectiva, articula la exposición de la DC: el contenido que aparece expuesto primero según la dialéctica del Ser y de la Esencia ha de ser re-puesto (Nuzzo, 2003, p. 173) en el interior del Concepto una vez que este ha emergido como resultado y fundamento de la Esencia. Desde aquí cada categoría que forma parte de la exposición de la DC puede ser considerada como parte de esta reposición; mientras que la ejecución de esta reposición no es sino el desarrollo de la libertad del concepto mismo, dirigida hacia el reconocimiento de su identidad en el ser-otro. Hegel se refiere a este desarrollo como realización del concepto (Realisierung des Begriffs), enfatizando con ello su carácter esencialmente activo, así como la raigambre ontológica de sus actos. Pues en este contexto "realizarse" no mienta sino que "el concepto ha de producir [herzustellen] [...] el libre ser-para-sí de su subjetividad" (Hegel, 2015, p. 132) y ello, precisamente, en la objetividad misma. Desde esta perspectiva, la realización del concepto se ofrece como un 208 | AlPHA No49 (DiCIEMBRe 2019) PÁGS. 207-223. ISSN 07 16-4254 
Fin subjetivo y razón instrumental en Ciencia de la lógica de Hegel

proceso en donde el concepto se hace efectivo en un mundo por medio de su producción. Este proceso va desde los grados más abstractos o formales hasta los más concretos y mediados. En estos últimos el concepto alcanza formas completas de mediación con la objetividad, vale decir, alcanza formas de reflexividad autoconsciente a partir de su presencia en lo objetivo.

Ahora bien, uno de los rasgos más particulares y fecundos del enfoque ofrecido por Hegel en la DC consiste en el modo en que esta se inscribe dentro del marco de una doctrina de categorías. En efecto, podemos considerar que las categorías tematizadas en la DC constituyen momentos de un proceso diversificado de constitución subjetiva o conceptual ${ }^{1}$ de la experiencia. O si se quiere: de configuración subjetiva de un "mundo". Pero el tipo de categorías que ofrece esta exposición excede el complejo de categorías y principios que Kant (2009) en su Analítica considera dotado de validez objetiva (vid. A96-A98; A154/B194-A162/B202), sin renunciar por ello a la pretensión de objetividad del concepto y sus determinaciones. Por este motivo es que la DC permite pensar fenómenos y problemas concretos, relativos al modo en que la racionalidad es efectiva en su mundo mediante su constante modificación. Precisamente, uno de estos fenómenos está constituido por lo que actualmente se conoce, en un sentido amplio, como racionalidad técnico-instrumental. En lo que sigue sostendremos que en el contexto de una teoría general de constitución subjetiva de la experiencia, el capítulo dedicado a la Teleología contiene una exposición y crítica de un momento "instrumental" del concepto. Lo característico del tratamiento hegeliano de este momento, denominado por Hegel como "fin subjetivo", es, como sugerimos, que el reconocimiento de la función específica y del factum mismo de un aspecto instrumental de la racionalidad se encuentre acompañado, como si se tratara de dos caras de una misma moneda, de una denuncia de sus insuficiencias y los peligros que acechan allí donde este momento se fija y adopta una posición universal.

Procederemos del siguiente modo: en primer lugar (1) abordaremos el objeto y la función específica del capítulo dedicado a la Teleología, para ello convendrá detenerse en algunas críticas dirigidas al capítulo, así como conectar su función con el problema general de la realización del concepto, lo que se expresa en el argumento de Hegel que a propósito de la antinomia mecanismo-finalidad. En segundo lugar, (2) nos referiremos en detalle a la actividad de realización del fin subjetivo, a sus limitaciones y al tipo de objetividad producido por dicha actividad. El argumento de Hegel expone este proceso en la forma de un silogismo, del que subrayaremos el término medio. Finalmente, (3)

\footnotetext{
${ }^{1}$ Debemos entender aquí "constitución subjetiva o conceptual" en un sentido general, pues la exposición del "ser del concepto" abarca i) modos formales de su autoconstitución (los tematizados en la sección Subjetividad); ii) modos externos de determinación (los tematizados en la sección Objetividad) y iii) modos inmanentes de (auto)determinación del sujeto en la objetividad (los tematizados en la sección Idea).
} 
procuraremos ofrecer una breve reflexión acerca del sentido general del capítulo en conexión con el problema general de un mundo producido por el hacer instrumental del concepto, así como proyectar el problema avistado al tránsito desde la Teleología a la sección Idea.

\section{OBJETO Y SENTIDO DEL CAPÍTULO TELEOLOGÍA}

Hegel sostiene que, luego del Mecanismo y el Quimismo (los dos primeros capítulos de la sección Objetividad), la "unidad esencial de los objetos está puesta [...] como diferente de su autosubsistencia [Selbständigkeit]; ella es el concepto subjetivo, pero puesto como referido en y para sí mismo a la objetividad", es decir, esta unidad esencial está puesta "como fin" (2015, p. 131). Que el fin (Zweck) sea la unidad esencial de los objetos, que además se haya diferenciado de ellos y al mismo tiempo los refiera, implica, entre otras cosas, que se trata de un fin subjetivo en el sentido de una reflexión externa a la objetividad. Por tanto, el objeto del capítulo Teleología consiste en una forma específica de fin propia de una reflexión subjetiva todavía limitada; no se trata en ella de un concepto general de teleología. Sin embargo, por tratarse de un tipo de finalidad, el fin subjetivo contiene ya la diferenciación general de esta respecto del mecanismo": "la Teleología tiene, en general, un principio más alto", pues ella expone "al concepto en su existencia [Existenz]" (2003, p. 157), sostiene Hegel. Para dilucidar la especificidad del fin subjetivo debemos considerar primero la diferenciación general en la que se inserta, y que hasta cierto punto anuncia por primera vez en la exposición de la Lógica.

Pues bien, el mecanismo constituye un reino de conexiones entre unidades autosubistentes que son exteriores e indiferentes. Estas unidades pueden, a lo sumo, formar un "agregado [Aggregat]" (Hegel, 2015 p. 134), una composición [Zusammensetzung], una "mezcla [Vermischung]" o un "cúmulo [Haufen]" (p. 133), pues en el mecanismo "la determinidad de un objeto es en cuanto ha sido puesta exteriormente en él, y ha sido puesta por algo otro" (p. 155). Hegel conecta este carácter exterior de las conexiones, así como de las leyes que las regulan, con el principio general del determinismo (p. 154): aquel que entiende lo verdadero en general según la forma de la ciega "necesidad de la naturaleza [Naturnotwendigkeit]" (p. 155). Frente a este principio, la teleología, en la medida en que ella expone el concepto en su existencia, exhibe el "principio de la libertad" o "autodeterminación" del concepto en cuanto "lo infinito y absoluto en y para sí" (p. 157). De este modo, y en cuanto opuesto a la libertad,

\footnotetext{
${ }^{2}$ Para seguir adecuadamente el argumento de Hegel debemos consignar que, en la Teleología, Hegel ofrece un concepto amplio de mecanismo que incluye en sí tanto al mecanismo como al quimismo en su especificidad; y ello en oposición al fin subjetivo: "lo que se expuso como quimismo viene ahora a ser tomado de consuno con el mecanismo, en la medida en que el fin es el concepto dentro de la libre existencia, y le está enfrentada en general la no-libertad del mismo" (2015, p. 155).

210 | Alpha No49 (DicIEMBRe 2019) PÁGS. 207-223. ISSN 07 16-4254
} 
el mecanismo expone el tipo de objetividad que dentro del concepto ha de ser considerada más inmediatamente como ser.

Si conectamos ahora el principio general de la teleología con el caso específico que se expone en el capítulo, entonces tenemos que en él se tematiza una forma de reflexividad libre del concepto que se encuentra referida "por entero" a un mundo que exhibe la solidez y autosubsistencia de lo objetivo, pero cuyas relaciones solo tienen el carácter de procesos mecánico-químicos. Esto podría formularse así: si en la objetividad inmediata del mecanismo el concepto está puesto como algo "extraño [fremd]" (Hegel, 2015, p. 133), lo que debe ocurrir en la Teleología es que él emerja en cuanto tal: existir objetivamente (i.e. en la objetividad) según su modo de ser: la autodeterminación. En este sentido es que, sistemáticamente, el capítulo opera un tránsito desde la objetividad inmediata del mecanismo hasta una objetividad que evidencia estar "completamente penetrada por el concepto" (p. 131), que contiene internamente el fin. Esta última es el reino de la idea, que se inicia con la vida: el concepto es allí adecuado a su existencia. Por eso allí se exponen formas plenas de automovimiento objetivo.

Puesto así el asunto, podemos decir que en la Teleología acontece una incardinación del fin en la objetividad; o en otras palabras: la tematización del fin subjetivo expone el proceso mediante el cual tal incardinación se lleva a cabo ${ }^{3}$.

\subsection{CRÍTICAS AL CAPÍTULO SOBRE LA TELEOLOGÍA}

Pero tan pronto como se consigue señalar el objeto y la función del capítulo surgen problemas. Hösle (1987) y Martin (2012) consideran injustificada la inclusión en la Objetividad del "concepto en su existencia". Para Martin esto interrumpiría la "continuidad del curso lógico" (pp. 410-411) entre mecanismo, quimismo y vida al intercalar acríticamente una forma reflexiva de acción -"actividad de fin autoconsciente" (p. 410)- antes de que hayan sido puestas sus condiciones lógicas necesarias. Pues si la idea expone una forma "animada de vida" (p. 407) que exhibe un fin interno, es desde allí que debería deducirse un caso específico de vida, a saber, la vida autoconsciente. De hecho esto es lo que ocurre en el paso de la vida al conocer. Por su parte, Hösle (1987) apunta al mismo asunto: ¿Cómo puede la vida representar un avance frente al espíritu que se pone fines? (p. 245). Pero su posición es más radical, ya que se inserta dentro de una crítica general a la sección Objetividad ${ }^{4}$.

\footnotetext{
${ }^{3}$ En este sentido Hegel sostiene: "Dado que el fin es el concepto puesto como un referirse en él mismo a la objetividad y superar por sí mismo su deficiencia, consistente en ser subjetivo, entonces la finalidad, en un comienzo externa, deviene por medio de la realización del fin interna $[\ldots]$ ", con lo que se llega "a la idea" (2015, p. 132).

4"Mecanismo, quimismo y vida no pertenecen a una filosofía fundamental entendida como ontología y lógica" (Hösle, 1987, p. 247). Hösle distingue diversos sentidos de teleología operativos en el texto y
} 
Es sin duda extraño que Hegel tematice al concepto en su existencia subjetiva antes de la vida y dentro de la sección Objetividad; y que después lo tematice como surgiendo de la vida, ahora cómo conocer que exhibe el impulso (Trieb) hacia lo verdadero y lo bueno. Pero también es difícil que no hubiese sido consciente de ello. ¿Por qué sería necesaria y justificada esta doble tematización de la existencia subjetiva del concepto? Lo primero es señalar que, respecto de este punto específico, los reparos de Martin y Hösle parecen encontrarse influidos por una especie de "historia natural", pues olvidan que:

Un malentendido capital [...] está en creer que el principio natural, o sea el inicio del que se ha partido en la evolución natural o en la historia del individuo que se forma a sí mismo, es lo verdadero y lo que en el concepto es lo primero. [...] Pero la filosofía no debe ser ninguna narración [Erzählung] de eso que sucede, sino un conocimiento de lo verdadero en lo que sucede, y desde lo verdadero es que ella debe además concebir eso que en la narración aparece como mero suceder [Geschehen] (Hegel, 2015, p. 22).

Por tanto, en la exposición del pensar puro -que ha de organizarse de acuerdo con lo que en el concepto es lo primero - la duplicación señalada podría ser legítimamente necesaria. Quisiéramos proponer la siguiente perspectiva para pensar tal necesidad: con vistas al tránsito desde la Objetividad a la Idea, surge la necesidad de tematizar una dimensión del concepto que, siendo libre, esté desprovista de una referencia a la verdad o el bien, ya que estos dos últimos contenidos ideales de la autorreferencia del concepto suponen la incardinación del fin en lo objetivo, primero como vida y luego como conocer. Esta dimensión reflexiva del concepto propia de la sección Objetividad tendría un carácter mediador entre el determinismo del mecanismo y la libertad del concepto. Se trata de un hacer libre que no obstante se agota en ser una referencia finita a la objetividad: el contenido del fin que busca cada vez el hacer libre no tiene el modo de ser del concepto, sino que tiene el modo de ser de aquello que está fuera de él; lo que orienta la actividad del concepto es por tanto una cosa para cuya consecución son requeridas, además, otras cosas. La determinación precisa de este hacer coincide con aquello que en términos amplios se entiende como razón técnico-instrumental o, como la llama Koch, una "filosofia de los artefactos" (2014, p. 218) destinados a su mera utilización. Un primer paso para argumentar en esta dirección se encuentra en la determinación del "fin subjetivo" en vista de la antinomia mecanismo-finalidad, cuyo contenido conforma un locus habitual para considerar el problema general implicado en el concepto de teleología,

considera que la categoría de "causa final" sí debería tener un lugar en la Lógica, pero "purgada" del "ropaje" que le da Hegel y ubicada en otro lugar, en ningún caso dentro de una sección destinada a la objetividad, ni tampoco como una forma subjetiva de reflexión.

212 | Alpha No49 (DiciemBre 2019) PÁGS. 207-223. ISSN 07 16-4254 
Fin subjetivo y razón instrumental en Ciencia de la lógica de Hegel

pero que adquiere en Hegel una función polémica (su discusión con Kant) y sistemática (el paso de la Objetividad a la Idea) central. Lo fundamental ahora será considerar la posición que el aspecto puramente instrumental del concepto tiene dentro de la antinomia señalada.

\subsection{FIN SUBJETIVO Y LA ANTINOMIA MECANISMO-FINALIDAD}

En el inicio del capítulo dedicado a la Teleología, Hegel aborda el problema de la antinomia necesidad-libertad usando también la formulación kantiana de la Crítica del Juicio: la antinomia mecanismo-finalidad. Como es de esperar, se trata de un concepto más general de teleología próximo al concepto de finalidad interna. Sin embargo, es posible derivar la posición que el fin subjetivo tiene dentro del problema en vista de esta antinomía.

Pues bien, Hegel señala en primer término que en el objeto en cuanto mecánico "no se manifiesta ninguna autodeterminación" (2015, p. 154). Por tanto, el dilema envuelto en la antinomia consiste en saber si es que hay algún tipo de causalidad en los objetos que manifieste una autodeterminación, o si, en definitiva, todo puede ser reducido a la necesidad propia del mecanismo, lo que conduciría al punto de vista del fatalismo (ibíd.) por cuanto sería imposible escapar del ciego destino de los objetos. ${ }^{5}$ Para Hegel esto equivale a preguntarse lo siguiente (véase p. 154): ¿Qué es aquello que tiene verdad en y para sí: o el modo de ser de lo mecánico, o el modo de ser de lo libre? La cuestión es clave si recordamos que la DC expone cómo es que el concepto se realiza en lo objetivo, lo que puede entenderse a partir de esta disyunción del siguiente modo: o la objetividad puede ser comprendida, en última instancia, según un modelo explicativo puramente mecánico, o en determinadas entidades ella exhibe un modo de ser que no solo requiere de un modelo explicativo de otra índole, sino que también muestra que la posibilidad de la libertad es efectiva y que además constituye la forma más elevada de realización de lo objetivo en general. Al respecto, Hegel busca mostrar que la finalidad es la verdad del mecanismo, y lo fundamental ahora es especificar en qué sentido -y hasta qué puntoesto lo es considerando la finalidad como fin subjetivo. ${ }^{6} \mathrm{Y}$ el primer obstáculo para ello viene dado por el descrédito de la teleología en la modernidad. El que ella sea considerada como algo "infantil [Läppischen]" (Hegel, 2015, p. 156) - un "asilo de la ignorancia" dice Spinoza en su Ética (1996, p. 71)-radica, precisamente, en pasar por alto el momento del fin subjetivo. El argumento teleológico convierte demasiado rápido la finalidad en la

\footnotetext{
${ }^{5}$ En este contexto resulta interesante notar que la categoría de destino (Schicksal) pertenece al Mecanismo, vid. Hegel, 2015, p. 141.

${ }^{6}$ Una interpretación más amplia, que excede el marco provisto por el capítulo dedicado a la Teleología (por tanto al concepto de fin subjetivo o finito), es realizada por Stekeler-Weithofer $(2009,2018)$, quien inscribe el problema presente en la Teleología dentro de una teoría general de la acción de corte pragmático.
} 
verdad de la objetividad: se toma de la naturaleza algún objeto contingente y se le asigna, no sin devoción (Frömmigkeit, Hegel, 2015, p. 155), un propósito subjetivo, propio de un entendimiento "extramundano". De ahí que Hegel subraye que el modelo explicativo del mecanismo parezca ser más objetivo y verdadero que el teleológico: simplemente aquel permanece en la explicación de las cosas mismas, en la investigación de la naturaleza. ${ }^{7}$

En este punto Hegel introduce la perspectiva de la actividad instrumental: es cierto que "allí donde se percibe finalidad se supone un entendimiento como autor de la misma" (p. 154); pero la Lógica niega que esto sea en virtud de un entendimiento extramundano que habría creado las cosas para un fin. El fin que se aprecia en los objetos de la naturaleza, en la contingencia del mundo en general, no lo es en virtud de su conexión con un propósito divino, sino en virtud de nuestra representación de lo que podemos hacer con ellos. Desde aquí podríamos formular la siguiente proposición: "El fin de las cosas es algo puesto por nosotros". En nuestro trato natural con las cosas, nosotros actuamos conforme a esta certeza implícita ${ }^{8}$.

Lo anterior anuncia indirectamente la solución de la antinomia: se trata de incorporar una mediación entre el objeto mecánico y el fin, esta mediación consiste en una actividad que transforma lo inmediato en conformidad con un fin representado. Debido a que la inmediatez objetiva de los procesos mecánicos son lo opuesto al concepto y este en cuanto "alma del estar [Dasein] objetivo" se enfrenta a ellos "en la forma de lo libre" (p. 30), los objetos se muestran para el concepto en tanto que fin como "lo que ha de ser determinado por su actividad" y, por tanto, como "lo nulo en y para s'́" (p. 131). Se aprecia entonces que Hegel informa de la emergencia finita, real, de un tipo de causalidad que no obedece al tipo de causalidad del mecanismo. O de otro modo: muestra cómo un determinado "objeto" introduce una relación distinta a la mecánica en objetos que se encuentran igualmente sometidos a las leyes del mecanismo; se trata de una

\footnotetext{
${ }^{7}$ La edición crítica (Gesammelte Werke) consigna que la consideración de la teleología como algo infantil se popularizó en la discusión filosófica gracias al siguiente aforismo de Schiller, aparecido en los Xenien de 1797: "El Teleólogo. ¡Cuánta veneración merece el creador del mundo! Con misericordia, al crear el alcornoque también encontró el corcho". Se aprecia que un objeto contingente como al alcornoque es puesto en una relación de finalidad con un fin igualmente contingente, a saber, tapar una botella, como si esta conexión fuera realizada por un entendimiento extramundano creador del alcornoque. Por tanto, se intenta declarar que en virtud de este entendimiento la naturaleza del alcornoque contiene la finalidad de convertirse en un corcho, cuestión que es absurda.

${ }^{8}$ Por tanto, el alcornoque sirve para hacer corchos con su corteza porque nosotros necesitamos tapar nuestras botellas de vino y buscamos un medio adecuado para ello en los objetos. El fin del alcornoque no puede entenderse sin referencia a nuestra subjetividad: su corteza es un corcho potencial solo para quien proyecta en él la finalidad de tapar una botella.

${ }^{9}$ Revelador es que Hegel diga que el concepto toma la posición de la "centralidad [Zentralität] de la esfera objetiva” (2015, p. 160), categoría que es propia de lo mecánico (pp. 143ss.), y que se había perdido en el Quimismo.
}

214 | AlPHA №49 (DiCIEMBRE 2019) PÁGS. 207-223. ISSN 07 16-4254 
causalidad que se sobrepone al mecanismo, lo conduce y lo trabaja ${ }^{10}$. Este peculiar "objeto" lo es en virtud de su pertenencia a la objetividad, y por tanto de su sometimiento relativo a las leyes del mecanismo, pero no es meramente un "objeto", es un sujeto, porque tiene la capacidad de representarse fines y buscar libremente su realización. Al adoptar libremente la posición de la centralidad, el fin subjetivo dispone al mundo como el medio (Mittel) de su realización.

Pues bien, si, como ha resultado de la sección Subjetividad, el silogismo es la forma completa de la automediación del concepto (Hegel, 2015, p. 126), entonces la mediación exigida entre su existencia para sí y la objetividad ha de acontecer según dicha forma, si bien en un principio de un modo externo. ${ }^{11}$ Es aquí donde Hegel posiciona el "silogismo de la referencia-de-fin [Zweckbeziehung]" (p. 159). El argumento es complejo y rico en matices. Nos enfocaremos en algunos aspectos puntuales, especialmente relacionados con la categoría de "medio".

\section{El MEDIO (MITTEL) COMO TÉRMINO MEDIO (MITTE) DEL SILOGISMO DE LA REFERENCIA DE FIN}

El silogismo del concepto adopta en la Teleología la forma específica del fin subjetivo. Su movimiento general busca concatenar el fin consigo mismo mediante la objetividad. Como se ha señalado, tal objetividad se encuentra, en principio, dada según la forma del mecanismo. Por tanto, se trata de modificar lo dado para darle una forma adecuada al fin: el fin debe "asumir su presuposición" (Hegel, 2015, p. 161). Durante esta modificación del contenido, el fin permanece como unidad esencial, como la "aspiración [Streben] e impulso [Trieb] esencial a ponerse exteriormente" (p. 160), i.e. regresar a sí mismo en lo otro. Esto implica que gracias a la intervención subjetiva, el contenido deja de tener la indiferencia propia de la determinidad mecánica, ya que es incorporada en la "referencia de fin", que Hegel caracteriza también usando una fórmula de la Esencia: se

\footnotetext{
${ }^{10}$ En tal sentido, sostiene Hegel: "La finalidad ahora se muestra por lo pronto como algo más alto en general; se muestra como un entendimiento que exteriormente determina la multiplicidad de los objetos a través de una unidad que es en y para sí, de modo que las determinidades indiferentes de los objetos devienen esenciales a través de esta referencia" (2015, p. 156).

${ }^{11}$ Hegel rescata del juicio reflexionante kantiano el que este provea un tránsito inmanente entre la singularidad de la intuición y la universalidad de la razón. Sin embargo, para Hegel el concepto en tanto fin conforma un "juicio objetivo" (una partición entre fin y objeto que acontece en la objetividad, así como un saber de algo que sucede en la objetividad misma, no en el mero modo del als-ob) y más aún, un silogismo: "Pero por eso, la referencia de fin no es un enjuiciar reflexionante que solo examina los objetos exteriores según una unidad como si un entendimiento la hubiese dado en referencia de nuestra facultad de conocer. Más bien ella es lo verdadero que es en y para sí [y] que objetivamente enjuicia y determina absolutamente la objetividad. Con ello, la referencia de fin es más que juicio, ella es el silogismo del concepto libre autosubsistente que se concatena silogísticamente consigo mismo a través de la objetividad" (p. 159).
} 
trata de una "referencia negativa a s'́", ahora del concepto. Según lo expuesto, es evidente que esta referencia involucra un "comportamiento negativo frente al objeto" (p. 161), pero más importante ahora es notar que en primer lugar se trata de un comportamiento que el concepto tiene respecto de sí mismo, comportamiento en el que se revela su naturaleza libre: el acto subjetivo de la resolución (Entschluss). Para entrar en un trato negativo con la objetividad, el concepto debe, primero, resolverse a salir de sí y realizarse en ella. Esto, negativamente, implica la renuncia a su propia subjetividad, la salida de su estado de inactividad. Pero positivamente implica la entrada en un proceso en el que el concepto i) se vincula con la objetividad y ii) refiere entre sí las diversas determinaciones de esta. Es el aspecto positivo el que nos conduce a la determinación concreta del medio.

El fin subjetivo es finito debido a que se encuentra fuera de los objetos en los cuales resuelve realizarse, dicha exterioridad lo torna tan contingente como el contenido que configura mediante su actividad. Debido a que tales objetos forman una totalidad que excede el alcance inmediato del fin y que además este no está en lo objetivo realizado (se trata de un proyecto), el fin no puede cumplirse en la objetividad de un modo inmediato. Por eso este requiere entrar indirectamente por medio de la inmediatez de lo objetivo, producir una clase de relación mecánica con los objetos subordinada a sí: usar lo inmediato para su propósito. Se requiere pues de un medio en el que él pueda ponerse inmediatamente y que al mismo tiempo pueda entrar en una relación objetiva de determinación con la objetividad. Por tanto, el medio expande el alcance del fin al manifestar una doble naturaleza: subjetiva por cuanto es la referencia inmediata del fin a lo objetivo, y objetiva, por cuanto él es un objeto dirigido por el fin que entra en una relación de determinación mecánica con otro objeto. ${ }^{12}$. Por este motivo Hubig (1995) ha visto en el capítulo acerca de la Teleología "una filosofía de la técnica in nuce" (p. 95) que ofrece una clave para pensar inmanentemente los procesos de innovación técnica. Según Hubig, Hegel descubre que nuestra "insuficiencia" o finitud exhibe en la relación fin-medio un "potencial de poder" (Hubig, 1995, p. 96), lo que da una primera señal de la dignidad que el medio tiene en cuanto primera forma de manifestación de lo racional en el mundo. En este sentido es que Hegel afirma que el medio es "algo más alto" que "los fines finitos y su finalidad externa" (2015, p. 166): "El arado es algo más lleno de honor de lo que inmediatamente lo son los placeres. [...] La herramienta se conserva, mientras los placeres inmediatos pasan y se olvidan" (p. 166).

Pero los medios no solo exhiben el poder de la razón, sino también su astucia (List, Hegel, 2015, pp. 165-166). Pues pese a que estos no son efímeros como puede serlo el disfrute contingente de un fin subjetivo, ellos se encuentran sometidos al destino de todo lo finito, a saber, el desgaste producido por la exposición a los procesos mecánicos. Por

\footnotetext{
${ }^{12}$ Hegel dirá: es subsumido por el fin y es subsumente de la exterioridad restante aún indeterminada, 2015, p. 163.

216 | AlPHA No49 (DiCIEMBRE 2019) PÁGS. 207-223. ISSN 07 16-4254
} 
tanto, lo que por un lado es violencia por parte del fin al hacer uso de su herramienta, por el otro lado es desgaste de esta y conservación del sujeto que se dirige a la consecución del fin. La razón es astuta porque en vez de exponerse a sí misma a este desgaste, se lo ahorra $^{13}$, con lo que difiere su desgaste sin por ello renunciar al cumplimiento de su fin y al disfrute del producto elaborado mediante la actividad ejecutada en el medio.

No obstante lo anterior, los medios también encubren un peligro, que se radicaliza allí donde la conexión fin finito-medio es totalizada. ¿A qué se refiere el peligro señalado? Para entrar en el aspecto crítico del argumento hegeliano conviene abordar directamente el silogismo del fin subjetivo. El medio es también el término medio del silogismo. Por tanto, la primera relación tematizada, a saber el fin subjetivo y el medio, consiste en la primera premisa de aquel. De acuerdo con esto, la segunda premisa sería la actividad del medio en el objeto aun exterior, donde debe cumplirse el fin. Esto daría como resultado la proposición conclusiva del producto (el fin cumplido): "lo universal es lo singular", o sea, "el fin es el objeto", se cumple en él ${ }^{14}$. Pues bien, Hegel denuncia que si bien el silogismo se cumple de este modo, el resultado no acontece del modo en que se lo espera, vale decir, no se consigue una verdadera realización del concepto en el fin cumplido.

Cabe distinguir i) el argumento "técnico" del texto y ii) el problema concreto que el argumento nos permite pensar.

\subsection{INSUFICIENCIA DE LA PROPOSICIÓN CONCLUSIVA DEL SILOGISMO: REPRODUCCIÓN INFINITA DEL MEDIO}

Fulda ha subrayado que una exigencia esencial del silogismo de la referencia de fin en general es que la actividad ejecutada en el medio no deba determinar al objeto "como algo exterior, sino que este objeto [el medio] debe coincidir por sí mismo con la unidad del concepto" (Fulda, 2003, p. 143). En tal sentido es que Hegel sostiene que el proceso teleológico es un "coincidir del concepto con sí mismo a través de sí mismo" (2015, p. 167), vale decir, él no se pierde en el desarrollo sino que permanece y se profundiza al configurar su propia naturaleza desde sí mismo. Esta exigencia no se

\footnotetext{
${ }^{13} \mathrm{Al}$ respecto cabe destacar la interpretación que Martin realiza de esta idea, tomando como un ejemplo particularmente revelador (pues se trata de un medio que se abstrae de intervenir en los procesos causalmecánicos que conduce) al termostato, el cual realiza su fin por medio de la interrupción (Unterlassung) de un proceso al alcanzar cierta temperatura (temperatura que operaría en principio como la representación subjetiva del fin): "Tal astucia es particularmente ingeniosa cuando un fin actúa por medio de la interrupción, pues con ello él no entra para nada en un intercambio recíproco que podría destruir a sus representantes [i.e. sus medios; S.M.] antes de su realización" (Martin, 2012, p. 414).

${ }^{14}$ Por tanto, el silogismo de la referencia de fin (A-B-E) tiene la siguiente forma:

Mayor: Fin subjetivo $\rightarrow$ Medio

Menor: Medio $\rightarrow$ Objeto externo

Proposición conclusiva: Fin subjetivo $\rightarrow$ Objeto
}

ALPHA № 49 (DiCIEMBRe 2019) PÁGS. 207-223. ISSN 07 16-4254| 217 
cumple cabalmente en el fin subjetivo pues el medio reproduce en el plano objetivo, cósico, la relación exterior del concepto en cuanto fin y el propio medio en cuanto primer objeto determinado; es decir: el fin es al objeto inmediato lo que el medio (el objeto determinado inmediatamente por el fin) es al objeto exterior en el que el fin debe cumplirse. Por tanto, en las premisas del silogismo se dan varias conexiones finalísticas que conservan el carácter exterior y todavía no realizado del fin. Lo que las premisas esperan (i.e. presuponen) del producto o conclusión es que el concepto evidencie que al conducir el proceso teleológico solo regresaba a su unidad, y esto es lo que mantiene la unidad del silogismo completo (la promesa de un regreso que exhiba la identidad de la conclusión con sus premisas). Es admisible, por tanto sostener que el proceso del fin subjetivo es exitoso en el sentido de que produce y realiza libremente cierto fin en el producto, pero presenta el siguiente problema: en su resultado no se constata una unificación positiva entre la singularidad del producto y la universalidad del fin, sino que el fin, al cumplirse en un objeto, permanece como exterior a él. En tal sentido es que se sostiene que la unidad entre el producto y sus determinidades "no puede deducirse desde la naturaleza específica del objeto" (p. 167), ya que ha sido puesta artificialmente en él, descansa en un propósito exterior que operaba como meta aún no verificada de un proceso exterior al producto mismo. El producto hace patente una diferencia entre el contenido del fin (el propósito subjetivo que busca realizarse en algo otro) y el contenido del objeto en el que el propósito se realiza (la cosa). Mientras que el primero es ideal y por tanto tiene la forma de lo libre, la referencia infinita del concepto a sí, el segundo es material y finito: se cumple solo en el agotamiento y en la referencia a un otro. El ejemplo de Hegel es muy claro (ver p. 169): una casa se muestra como fin frente a las herramientas utilizadas para su construcción, pero su efectividad (lo que en el argumento se denomina "determinidades del producto"), i.e. su techo, sus vigas, etc., solo cumple su fin al someterse a procesos de uso y desgaste como la lluvia, los terremotos o una fiesta especialmente desordenada. Además, esta casa, que es fin frente al martillo, no lo es frente a nuestra necesidad de habitar en ella, de hacer una fiesta, o frente a la empresa inmobiliaria que la construye como medio para obtener plusvalía. Por esto Hegel concluye enfáticamente que "el producto del hacer conforme a fin no es otra cosa que un objeto determinado por un fin exterior a él; por lo tanto es lo mismo que el medio. En consecuencia, en tal producto se ha llegado solo a un medio, no a un fin cumplido, o el fin no ha alcanzado en él verdadera objetividad" (p. 169).

$\mathrm{Si}$ atendemos ahora a la estructura silogística del argumento entonces apreciamos que la insuficiencia que muestra el producto en tanto conclusión hace que se desfonden también las premisas: como se apuntó, lo que mantenía la unidad de las premisas era la identidad presupuesta entre concepto (el fin) y la objetividad, pero al mostrarse que, al llegar al producto, el fin más bien "se fuga" (solo queda un artefacto hecho con finalidad, pero no la existencia del fin en y para sí), la exterioridad entre el medio y los extremos 218 | AlpHA №49 (DiCIEMBRE 2019) PÁGS. 207-223. ISSN 07 16-4254 
pierde aquello que le "prestaba" su concreción, lo que redunda en un desborde de las premisas hacia un mal infinito doble: la verdad de la primera premisa es que el fin solo puede ponerse como medio por otro medio, etc.; la verdad de la segunda premisa es que el producto es un medio para otro fin, y este fin será nuevamente un medio, etc. Este hecho, que Stekeler-Weithofer entiende como la base de una historia de la técnica ${ }^{15}$, revela que el término medio no contenía verdaderamente los extremos, sino que era una identidad meramente presupuesta, aseverada desde la posición exterior del fin subjetivo: "el silogismo tiene [...] el defecto del silogismo formal en general, [a saber] que las referencias en las que él consiste no son ellas mismas proposiciones conclusivas o mediaciones; ellas mismas más bien presuponen ya la proposición conclusiva hacia cuya producción, en cuanto medio, tendrían que servir" (Hegel, 2015, p. 168). Por tanto, el fin no se realiza en los medios, no es la existencia conceptual o libre de estos; no constituye pues una forma de automovimiento en la objetividad, sino el hacer de una subjetividad formal.

\subsection{EL MUNDO COMO UN CÚMULO DE MEDIOS}

Como hemos adelantado, consideramos que el resultado alcanzado permite pensar más concretamente el peligro potencial de la determinación meramente instrumental de los medios. A propósito de la antinomia entre mecanismo y teleología, Hegel (ver p. 155) ya había notado que ambos términos exhiben una tendencia a la totalidad. Si la absolutización del mecanismo conduce al fisicalismo y, en última instancia, al fatalismo, tendríamos que considerar qué es lo que ocurre con la tendencia infinita del fin subjetivo, tal como se ha caracterizado. El avance de esta forma limitada de actividad del espíritu convierte al mundo en un cúmulo de medios disponibles, sometidos al uso y al desgaste sin más principio que el de una finalidad exterior que, o bien permanece abstracta e indefinida, o bien es una determinación arbitraria, particular que se absolutiza de un modo, si se quiere, ideológico. Pese a funcionar, este mundo creado por el hacer instrumental del espíritu no exhibe la realización del modo de ser del concepto, vale decir, la libertad. Más bien se revela como lo contrario de esta, cuestión que Hubig (1995) subraya con el concepto de "señorío de los medios" (p. 97): se trata de un proceder del espíritu que avanza de medio en medio hasta configurar la realidad, natural y espiritual, como un enorme engranaje de referencias instrumentales, una "máquina del mundo" como le ha denominado Duque (1998, p. 730). En este enorme engranaje el propio espíritu se ve envuelto como un medio más, cuestión que se constata p.ej. en el hecho de que cada vez dependemos más, y más sofisticadamente, de los medios y productos que

\footnotetext{
${ }^{15}$ En tal sentido es que Stekeler-Weithofer habla de una "historia del desarrollo y del uso de técnicas" (s.f., en línea).
} 
deberían liberarnos, que ellos nos exigen cada vez más una mayor especialización para manipularlos, conservarlos o repararlos, y que a su vez presentan problemas o consecuencias de cada vez mayor impacto y más difícil manejo o previsión ${ }^{16}$. Todo este engranaje opera a costa del desgaste tanto de la vida natural como de la vida del espíritu, devenido un mecanismo espiritual. Fulda (2003) condensa esta paradoja en vista de la antinomia entre mecanismo y finalidad: "mecanismo y finalidad externa se relacionan entre sí no solo como si esta fuera la verdad última del mecanismo [...]. Ambos son también el uno la verdad del otro" (p. 145).

\section{CONSIDERACIONES FINALES}

Para concluir quisiéramos, en primer lugar, sintetizar algunos de los puntos mencionados en el texto a partir de la siguiente pregunta: ¿Qué sentido puede tener la posición del capítulo Teleología interpretado como una lógica de la actividad instrumental del concepto? Además, en segundo lugar, buscaremos proyectar brevemente el sentido del capítulo en vista del tránsito a la Idea de la Vida, lo que puede considerarse como un delineamiento de coordenadas para una etapa siguiente de la investigación.

Pues bien, la tematización del fin subjetivo como una forma de reflexividad subjetiva que todavía es exterior a la Idea (i.e. es anterior a la Vida y forma parte de la Objetividad) exhibe, desde la perspectiva que hemos procurado ofrecer, la siguiente especificidad: Hegel busca ofrecer allí una determinación categorial que exhiba tanto un aspecto propio de la determinidad que atraviesa la Objetividad, vale decir, del modo de ser del mecanismo, como un aspecto que exhiba el modo de ser del concepto, lo libre. En tal sentido, la categoría de "fin subjetivo" presenta, ciertamente, un hacer libre del concepto, pero este hacer se encuentra - en su emergencia- enteramente referido a la objetividad en cuanto tal, es decir, considera a la cosa en cuanto cosa como contenido del fin. Por este motivo, el fin subjetivo instaura cierta dinámica objetiva en la que él mismo se ve envuelto, de modo que el sentido de la incorporación de tal categoría en la Objetividad podría ser determinado más precisamente de este modo: Hegel advierte que existe un tipo de hacer del concepto que, considerado en cuanto tal, bien podría ser absorbido por la dinámica

\footnotetext{
${ }^{16}$ Tomamos estos tres aspectos del análisis de Hubig (1995, pp. 92-93), quien conecta los procesos de innovación técnica con la cada vez más desarrollada automatización de la tecnología. Ahora, si tal como sostiene Harvey (2007) "la teoría neoliberal del cambio tecnológico descansa en la fuerza coercitiva de la competencia para impulsar la búsqueda de nuevos productos, de nuevos métodos de producción y de nuevas formas organizativas", hasta el punto de producir cierta "creencia obsesiva de que existe una componenda tecnológica para todos y cada uno de los problemas que puedan plantearse" (p. 78), entonces podríamos agregar un aspecto más a los destacados por Hubig, a saber, que el proceso de conversión del mundo en un cúmulo de medios se encuentra estrechamente ligado a una progresiva mercantilización de la vida (la vida como medio para la acumulación) tal como se ha venido dando en la era neoliberal.
}

220 | AlPHA No49 (DiCIEMBRe 2019) PÁGS. 207-223. ISSN 07 16-4254 
propia de la objetividad. Dicho de otro modo, como mero fin subjetivo, lo libre puede transformarse en un proceso con rasgos mecánicos; y aún más: el hacer del concepto puede devenir una actividad mecanizadora de la vida, incluso a costa de sacrificar el carácter libre de su esencia. Pensemos desde aquí en una figura concreta: el trabajo, la instancia de aplicación del saber en sus diversos grados, puede ser medio para la realización del ser humano en la naturaleza, pero también puede ser la instancia de enajenación de su libertad, o una actividad que destruye su propia vida, al igual que la vida en general.

Con la Teleología Hegel distingue conceptualmente esta dimensión puramente instrumental de la actividad del espíritu. Con ello, da cuenta de su realidad efectiva, de la mediación necesaria que ella representa para un mundo en el que se desarrolla una forma de vida libre. Pero al mismo tiempo Hegel denuncia los peligros de convertir a la actividad instrumental en el principio que determina la actividad del espíritu. Sin duda hoy experimentamos el factum de la dimensión instrumental de la racionalidad de un modo radicalizado: en el contexto tecnológico actual, la aparición de la vida en general se encuentra mediada por una multiplicidad inconmensurable de referencias instrumentales. Se ha vuelto evidente que la aplicación del saber científico es eficiente para la transformación de la naturaleza y la consecución de múltiples fines, pero también dicha aplicación ha mostrado operar de un modo independiente al hecho de si la consecución de tales fines (más precisamente: la referencia infinita de medios) contribuye o no a la construcción de un mundo mejor, mostrándose muchas veces indiferente ante interrogantes relacionados con el sentido de su propia actividad.

Por tanto, la posición del "fin subjetivo" frente a la Vida apunta al hecho que la actividad instrumental puede encontrarse o no asumida (aufgehoben) en una forma de fin más alta, pero de todas formas mediará la objetividad inmediata. En la exposición lógica, Hegel dará un sentido racional a esta mediación necesaria, lo que requiere de la diferenciación entre la forma de finalidad finita presente en el capítulo Teleología y una forma interna de fin. Esto puede iluminarse si enfatizamos la limitación del "fin subjetivo".

Sabemos ya que el núcleo de la deficiencia del "fin subjetivo" radica en la posición exterior del fin, en el carácter exterior de la reflexión subjetiva. Sin embargo, la finitud de esta reflexión no lo es según su forma, pues esta reflexión ya tiene la forma del silogismo, y presupone la automediación de este. Más bien -como apuntamos ya- tal finitud se refiere al contenido en el que el fin busca realizarse: el fin subjetivo busca su realización en la objetividad del mundo, pero considera el contenido de su autoreferencia según el modo de lo cósico. Por tal motivo su intento de realización nunca es satisfactorio, nunca regresa a sí mismo en este proceso de transformación y desgaste de lo objetivo. Lo libre solo se muestra allí ex negativo (ver Hubig, 1995, p. 97), vale decir, como la negación de la exterioridad del fin y como la negación de cada determinación del contenido. En términos sistemáticos, sería este trabajo negativo lo que llevaría a cabo la incardinación del fin que anteriormente hemos señalado como tarea mediadora del capítulo. Pues bien, 
es aquí donde se revela el sentido del tránsito a la Idea, sección que comienza con la Idea de la Vida. Quisiéramos finalizar apuntando la dirección que podría tomar el argumento de Hegel en conexión con nuestro problema.

Stekeler-Weithofer $(2009$, p. 115) ha detectado que en la Teleología hace su entrada el silogismo de la acción racional en general, si bien allí esta forma libre aparece todavía referida a un contenido finito y exterior, y el propio concepto aparece como una posición parcial que adopta la forma de la universalidad. Esto cambiará primero con la tematización de lo orgánico en la Vida, donde comienza la exposición de un automovimiento objetivo. Sin embargo, tal vez lo decisivo para el problema localizado venga dado por la incorporación, en la Idea del Conocer, de la categoría de bien como contenido ideal de la acción racional ${ }^{17}$. Pues, más allá de las críticas que resulten de su tematización específica, la idea de bien en cuanto fin no tan solo debería conducir los procesos mecánico-químicos (al modo del alma en el cuerpo vivo), sino también debería orientar e integrar la actividad instrumental del espíritu mediante un criterio que no se agota en corresponder a una cosa, sino que corresponde a lo que el concepto exige de sí mismo cada vez para realizarse y reconocerse en la realidad. El sentido y las condiciones de esta realización, la verificación de la convergencia entre el principio o criterio de realización y el hacer específico que se dirige hacia ella, así como la determinación suficiente de los momentos implicados en el proceso de realización, son encomendados por Hegel a una forma de saber que no se limita a un modelo explicativo mecánico, ni tampoco se encuentra subordinada a criterios de aplicación instrumental. Los rasgos básicos de esta forma de saber, así como su proceder metódico, son formulados dentro de la Lógica en la sección dedicada a la Idea Absoluta en conexión con una teoría de la autoconsciencia que exhibe una raigambre histórica e intersubjetiva.

\section{OBRAS CITADAS}

Duque, Félix (1998). Historia de la Filosofía Moderna. La era de la crítica. Madrid: Akal. Fulda, Hans Friedrich (2003). "Von äußeren Teleologie zur inneren". En: A.F. Koch und A. Obernauer (eds.), Der Begriff als die Wahrheit. Zum Anspruch der Hegelschen “Subjektiven Logik”, Paderborn: Schöningh, 135-150.

\footnotetext{
${ }^{17}$ Ya en la Teleología Hegel anuncia la necesidad de conectar la deficiencia del fin finito con la idea de bien como principio: "Pero si, por lo demás, este [el contenido del fin] es finito e insignificante, entonces contradice él eso que él debe ser [a saber, el fin], pues el fin es, [según] su forma, una totalidad infinita en sí - particularmente si se supone que el actuar que es efectivo según fines es una entendimiento y una voluntad absolutos" (2015, p. 155). Por lo demás, Hegel incorpora expresamente el silogismo de le referencia de fin es el capítulo sobre la idea de bien, ahora como "silogismo de la acción" (pp. 233-234) y el argumento se dirige, precisamente, a mostrar como este se realiza como una totalidad en sí misma.
}

222 | AlPHA No49 (DiCIEMBRe 2019) PÁGS. 207-223. ISSN 07 16-4254 
Fin subjetivo y razón instrumental en Ciencia de la lógica de Hegel

Harvey, David (2007). Breve historia del Neoliberalismo, trad. A. Varela, Madrid: Akal. Hegel, Georg Wilhelm Friedrich (2015). Wissenschaft der Logik. Die Lehre vom Begriff (1816), Philosophische Bibliothek, Band 377 (edición según las Gesammelte Werke [=GW]; se cita de acuerdo a la paginación de GW 12), Hamburg: Meiner, 2003. Trad.: Ciencia de la Lógica. I. La Lógica subjetiva (1816), trad. F. Duque, Madrid: Abada.

Hösle, Vittorio (1987). Hegels System. Der Idealismus der Subjektivität und das Problem der Intersubjektivität, I. Hamburg: Meiner.

Hubig, Christoph (1995). 'Die Dialektik der Mittel. Zur 'immanenten Logik' technischer Innovationsprozesse". En: H. Lenk y H. Poser (eds.) Neue RealitätenHerausforderung der Philosophie, Berlin: Akademie, 87-101.

Kant, Immanuel. (2009). Kritik der reinen Vernunft, Philosophische Bibliothek, Band 505, Hamburg: Meiner.

Koch, Anton Friedrich (2015). "Subjektivität und Objektivität: Die Unterscheidung des Begriffs". En: C. Wirsing, A. F. Koch (eds.), Hegel-200 Jahre Wissenschaft der Logik, Hamburg: Meiner, 209-242.

Martin, Christian (2012). Ontologie der Selbstbestimmung. Eine operationale Rekonstruktion von Hegels "Wissenschaft der Logik”, Tübingen: Mohr Siebeck.

Nuzzo, Angelica (1995). "Zur logischen Bestimmung des Ontologischen Gottesbeweis. Bemerkungen zum Begriff der Existenz im Anschluß an Hegel”. Revista HegelStudien $\mathrm{N}^{\mathrm{o}} 30,105-120$.

— (2003). 'Existenz 'im Begriff' und Existenz 'außer dem Begriff'. Die Objektivität von Hegels 'subjektiver Logik"'. En: A.F. Koch y A. Obernauer (eds.), Der Begriff als die Wahrheit. Zum Anspruch der Hegelschen "Subjektiven Logik”. Padeborn: Schöningh, 171-187.

Schäfer, Rainer (2001) Die Dialektik und ihre besonderen Formen in Hegels Logik. Entwicklungsgeschichtliche und systematische Untersuchungen, Hamburg: Meiner.

Spinoza, Benedictus (1996). Ética. (trad. Vidal Peña). Madrid: Alianza.

Stekeler-Weithofer, Pirmin (sin fecha). "Hegels Ortsbestimmung des zweckorientierten Handelns und der praktische Schluß als Formbedingung freier Entscheidung". Disponible en: http://hegel.net/werkstatt/personen/stekeler/zweckorientertes_ handeln.htm [Consulta: 2 abril 2018].

(2009). "Teleologie als Organisationsprinzip. Zu Hegels Kritik an Kants (Krypto)Physicalismus". En. B. Sandkaulen, et al. (eds.), Gestalten des Bewusstseins. Genealogisches Denken im Kontext Hegels, Hamburg: Meiner, 102-134. 